\title{
Findings in [99mTC]MAA SPECT/CT in the diagnosis and follow-up of pulmonary embolism after infection by SARS-CoV-2 (COVID-19)
}

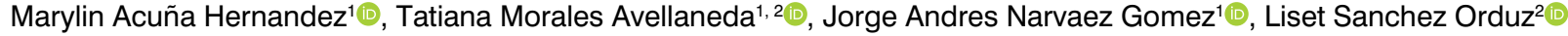 \\ 'Universidad AutóNoma De Bucaramanga, Santander, Colombia \\ ${ }^{2}$ Spect Medicina Nuclear S.A.S, Centro medico carlos ardila Lulle, Bucaramanga, Colombia
}

[Received 5 III 2021; Accepted 15 III 2021]

\begin{abstract}
SARS-CoV-2 (COVID-19) infection is a current public health problem that has been shown to cause multiple complications, including pulmonary thromboembolism. The first presented case is a 59-year-old woman with a history of COPD, paroxysmal atrial fibrillation and COVID-19 infection in September 2020, consultation in December 2020 for atypical chest pain with suspected PE, AngioCT of pulmonary vessels was performed negative for emboli, subsequently [99mTc]Tc MAA SPECT/CT was indicated with a report of multiple triangular defects concerning acute pulmonary thromboembolism. A second case is a 70-year-old man with a history of dyslipidaemia, presented COVID-19 infection in September 2020 with a complication of PE with involvement of the left pulmonary artery, followed by [99mTc]Tc MAA SPECT/CT report multiple triangular and not triangles defects concerning pulmonary thromboembolism with signs of reperfusion.
\end{abstract}

KEY words: pulmonary embolism; coronavirus infections; ventilation-perfusion scan; diagnosis

Nucl Med Rev 2021; 24, 2: 120-121

SARS-CoV-2 (COVID-19) infection is a current public health problem that has been shown multiple complications, including pulmonary embolism. This study presents the case of a 59-yearold woman with a history of COPD, paroxysmal atrial fibrillation and COVID-19 infection in September 2020, consultation in December 2020 for atypical chest pain, ruling out cardiac, vascular and infectious ischemic causes, finding in TT echocardiogram sign of precapillary pulmonary hypertension. PE was suspected and therefore negative AngioCT of pulmonary vessels was indicated, although the clinical doubt persisted, starting anticoagulation and 7 days later $\left.{ }^{99 \mathrm{~m}} \mathrm{Tc}\right]$ Tc MAA planar images and SPECT/CT were indicated (Fig. 1 A, B) evidence of triangular defects in the left upper lobe apicoposterior segment, upper and lower lingular and non-triangular hypoperfusion areas of the lower left lobe posterior basal

Correspondence to: Marylin Acuña Hernandez

Universidad AutóNoma De Bucaramanga, Santander, Colombia

e-mail: macuna766@unab.edu.co segment and upper right lobe apical and lateral segments without related morphological alterations, making a diagnosis of acute PE according to MSKCC Q-SPECT/CT Criteria [1] with signs of reperfusion.

The second case is a 70-year-old man with a history of dyslipidaemia, presented COVID-19 infection in September 2020 during hospitalization. AngioCT of pulmonary vessels was made evidence pulmonary embolisms in the left pulmonary artery, anticoagulation was started and in December 2020 requested control with [ ${ }^{99 \mathrm{~m} T C}$ ] TC MAA planar images and SPECT/CT (Fig. 2 A-B) triangular defect in right upper lobe apical segment and non-triangular upper left lobe apicoposterior segment without morphological alterations concerning PE according to MSKCC Q-SPECT/CT Criteria [1] with signs of reperfusion.

It is currently known that viral infections, especially by SARS-CoV-2 (COVID-19), can predispose to pulmonary thromboembolic events, the active systemic inflammatory cascade that leads to a procoagulant state due to endothelial dysfunction and hypoxia, in the case of pulmonary involvement generates bronchoalveolar haemostasis with a generation of microthrombi [2, 3]. 


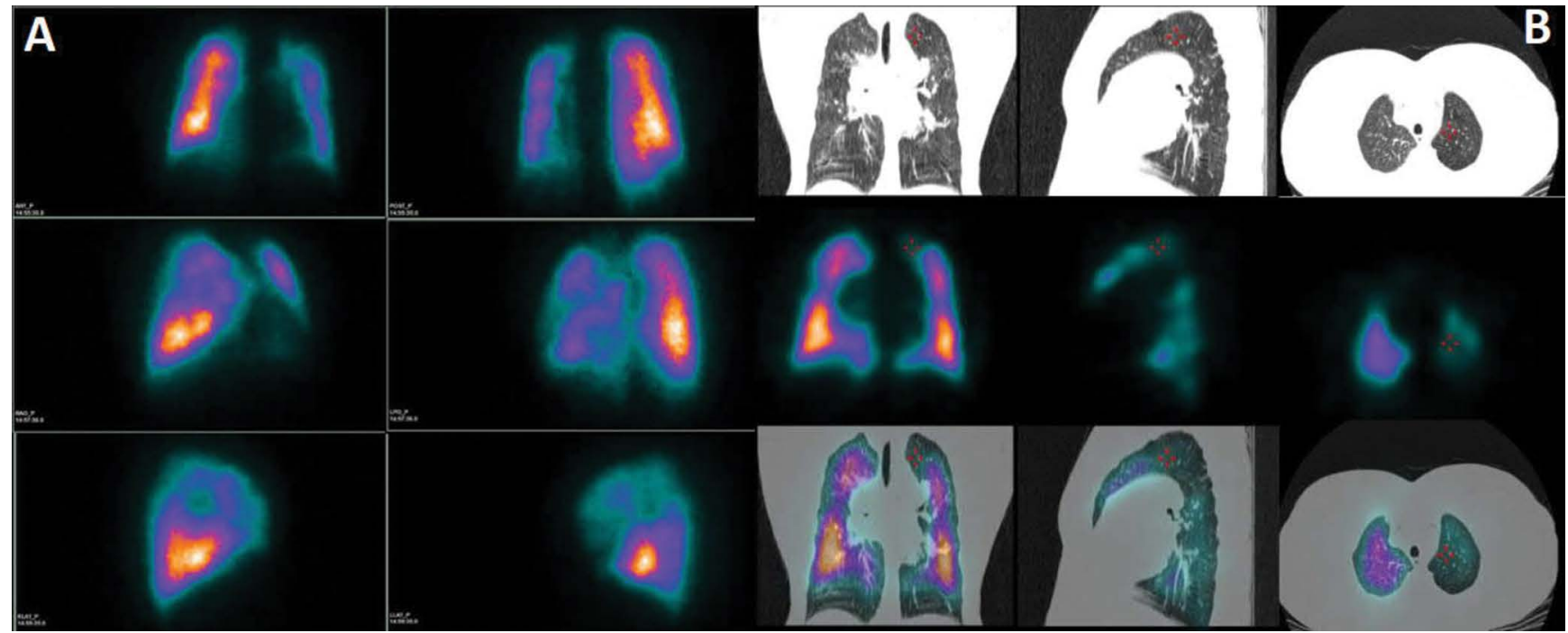

Figure 1. Triangular defects in the left upper lobe apicoposterior segment, upper and lower lingular and non-triangular hypoperfusion areas of the lower left lobe posterior basal segment and upper right lobe apical and lateral segments without related morphological alterations

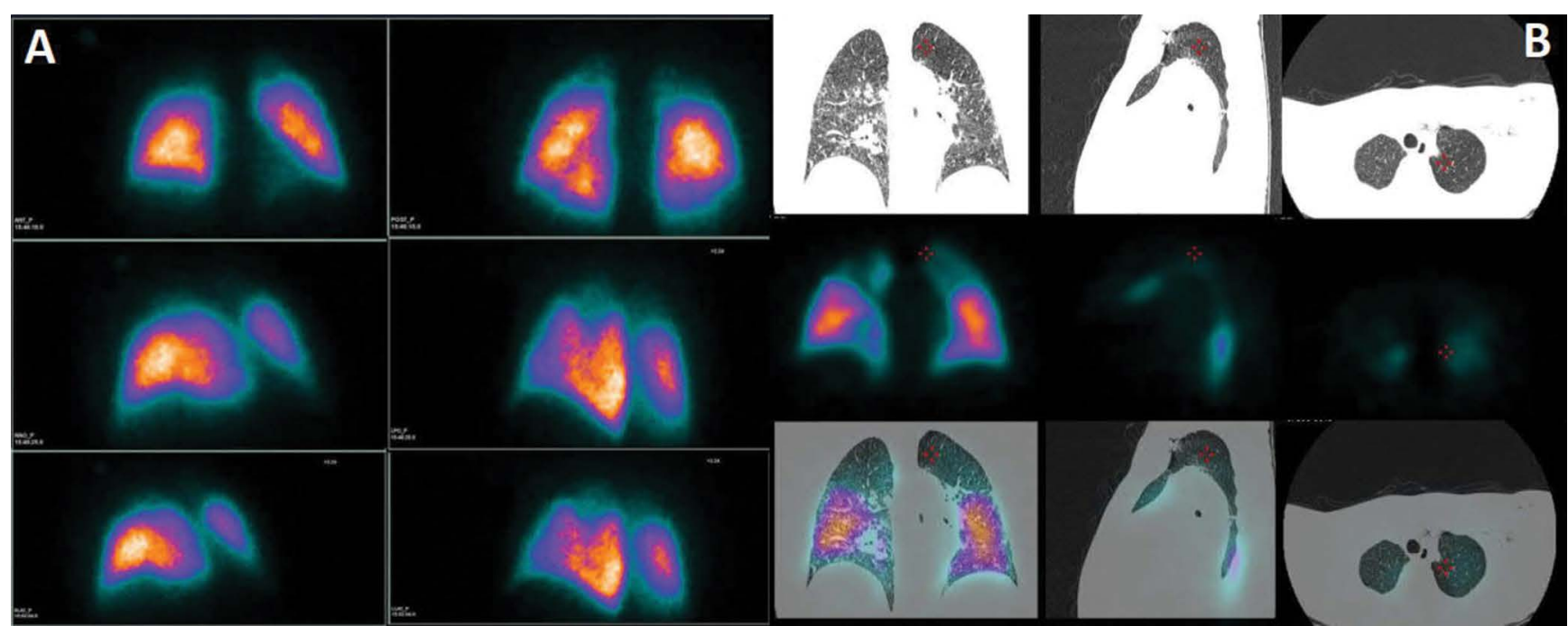

Figure 2. Triangular defect in right upper lobe apical segment and non-triangular upper left lobe apicoposterior segment without morphological alterations

In the same way, it has been described that the affectation in these individuals is due to pulmonary artery thrombosis secondary to the phenomena referred to in the previous paragraph that generates severe pulmonary inflammation that triggers a hypercoagulable state instead of serious thromboembolism, venous aetiology [3].

\section{Conflict of interest}

The authors declare that they do not have any conflict of interest.

\section{References}

1. Lu Y, Macapinlac HA. Perfusion SPECT/CT to diagnose pulmonary embolism during COVID-19 pandemic. Eur J Nucl Med Mol Imaging. 2020; 47(9): 2064-2065, doi: 10.1007/s00259-020-04851-6, indexed in Pubmed: 32383092.

2. Sakr $Y$, Giovini M, Leone $M$, et al. Pulmonary embolism in patients with coronavirus disease-2019 (COVID-19) pneumonia: a narrative review. Ann Intensive Care. 2020; 10(1): 124, doi: 10.1186/s13613-020-00741-0, indexed in Pubmed: 32936400.

3. Cavagna E, Muratore F, Ferrari F. Pulmonary Thromboembolism in COVID-19: Venous Thromboembolism or Arterial Thrombosis? Radiol Cardiothorac Imaging. 2020; 2(4): e200289, doi: 10.1148/ryct.2020200289, indexed in Pubmed: 33778609 